\title{
A Dynamic Model of Gambling Addiction with Social Costs: Theory and Policy Solutions
}

\author{
Nazaria Solferino ${ }^{1}$, Leonardo Becchetti², Maria Elisabetta Tessitore ${ }^{2}$ \\ ${ }^{1}$ University of Tor Vergata, Rome, Italy \\ ${ }^{2}$ Department of Economics, University of Tor Vergata, Rome, Italy \\ Email: nazaria.solferino@uniroma2.it
}

How to cite this paper: Solferino, N., Becchetti, L. and Tessitore, M.E. (2018) A Dynamic Model of Gambling Addiction with Social Costs: Theory and Policy Solutions. Modern Economy, 9, 2039-2051. https://doi.org/10.4236/me.2018.912127

Received: November 6, 2018

Accepted: December 10, 2018

Published: December 13, 2018

Copyright (C) 2018 by authors and Scientific Research Publishing Inc. This work is licensed under the Creative Commons Attribution International License (CC BY 4.0).

http://creativecommons.org/licenses/by/4.0/

\begin{abstract}
We investigate the effects of gambling addiction in a dynamic model in which wellbeing crucially depends on the accumulation of relational goods which is weakened by the consumption of gambling. We outline conditions under which gambling may become addictive leading to a suboptimal equilibrium from a social point of view. We examine the relative effectiveness of different policy solutions (tax on gambling, gambling restrictions) in bridging the distance between the equilibrium without intervention and social optimum.
\end{abstract}

\section{Keywords}

Addiction, Gambling, Social Costs

\section{Introduction}

In the neuroscience literature [1] [2] Pathological Gambling (from now on PG) is considered as a form of addiction (and violation of the rationality paradigm) since the pathological gambler "takes pains to engage in an activity that transparently has negative expected returns if utility varies positively with money. She also, typically, spends further resources on commitment devices designed to interfere with her gambling" [2]. A compulsive, or pathological, gambler is someone who is unable to resist his or her impulses to gamble. This leads to severe personal and, or, social consequences. The urge to gamble becomes so great that tension can only be relieved by more gambling. The association between PG and classic (substance) addiction is therefore particularly strong and, consequently, the more general addiction literature may be an important background to study the PG phenomenon.

The origins of the economic literature on addiction trace back to the 
pioneering contributions of Stigler and Becker [3], Iannacone [4] and Becker and Murphy [5] who actually start from the consideration that addiction and economic rationality are not necessarily mutually exclusive.

In Becker and Murphy [5] rational agents, despite recognizing the addictive nature of their choices, still perform them because future addiction makes expected gains from gambling higher than their costs. Nevertheless, paying the full price of addictive consumption goods acts as a deterrent since it represents not only a current monetary cost but also a permanent expected decrease in wealth due to the future expected gambling expenditure generated by addiction.

Gul and Pesendorfer [6] prooose a model where consumption of the addictive good is a tempting choice that erodes self-control in the future. Both papers agree with Becker and Murphy [5] and document the effectiveness of interventions based on raising the price of addictive goods such as smoking, alcohol and heroin when they successfully reduce the demand for them.

In the past two decades there has been a significant increase in academic interest concerning gambling behavior and, with it, the economic and social impacts of legal gambling, as a special case of the addiction literature. However, to our knowledge, only a relatively small portion of gambling research has been performed in economic disciplines [7]. In this respect Bardsley [8] devises a dynamic game in continuous time between the seller and the consumer of an addictive substance while Hartley and Farrell [9] investigate the ability of the expected utility theory to account simultaneously for gambling and insurance.

We aim to contribute to this literature with a model of rational addiction applied to gambling by taking into account the different effects that addiction has on both the consumption of the addictive good and, at the same time, the consumption of relational goods/stimulus goods. Based on this theoretical background, our paper also analyzes the relative effectiveness of different policy remedies to the gambling problem and, in doing so, it deals with the well-known and general issue of paternalism and of the boundaries between what should be legal and what not. With this respect our work is related to some recent papers specifically analyzing the role of the state and gambling legalization. An example is the contribution of Walker and Jackso (2011) [10] who examine the overall impact of legalized casino gambling on government revenues. The authors show that, despite the economic benefits that casinos provide in terms of additional tax revenues, a relevant amount of social costs is also generated. According to Layton and Worthington [11] the United States successfully extracted more than $9.78 \%$ of total state taxation from gambling. Unfortunately, these revenues are not produced without some undesirable socio-economic problems such as the relative detriment of low income individuals and the increasing need for public support programs [12] [13] [14] [15]. Furthermore, there is evidence that certain forms of gambling may create compulsive gambling and major addiction, attract criminal elements and foster corruption [16]. Treating addicts, supporting families in difficulty, fighting crime structured around PG, are activities that generate high financial costs which are mainly supported by local structures 
(municipalities, local health authorities, police forces), but the same activities may also cause a decrease in the national budget as the epidemic progresse. In the second part of our paper we aim to take into account the role of government against the adverse effects of gambling. In this respect, we focus on two possible alternative government policies. The first one is based on a price increase while the second one on limitations to gambling opportunities. We found that the second alternative can lead to more efficient results when the government is not myopic and takes into account social losses from gambling.

The paper is organized as follows: in Section 2 we describe the main features of our model and discuss its equilibrium characteristics. In Section 3 we model the social costs of gambling for "passive" members in the same household. In Section 4 we compare the two possible alternative policies and we argue how the policymaker trade-off between gambling revenues and wellbeing may be afgected in favour of the latter by the bottom-up action of citizens. Section 5 concludes.

\section{The Dynamic Model of Gambling Addiction}

Our model borrows extensively from Dragone et al. (2016) [17] and assumes that a rational agent chooses, given her budget constraint, how much to consume of:

1) a good $s$ which creates addiction (i.e. gambling with slot machines) 2;

2) a relational good $r$;

3) a standard composite numerary consumption good $q$ with unit price.

From a dynamic point of view the agent's utility also depends on a variable a representing addiction to gambling and the accumulation of well-being from the good $w$ depending on past and current consumption of (investment in) $r$ and decreasing over time with the consumption gambling. Assuming that both $a(t)$ and $w(t)$ have the same positive rate of decay $\delta$ we have that a evolves over time depending on current gambling choices and addiction from gambling activity accumulated in the past:

$$
\dot{a}(t)=s(t)-\delta a(t)
$$

while $w$ is ruled by:

$$
\dot{w}(t)=r(t)-\delta w(t)-\varepsilon s(t) .
$$

where $\varepsilon$ is a positive constant.

In particular, we analyze the different preferences of individuals characterized by greater attachment to the pleasure of gambling, considering the total utility as a quasi-linear weighted sum of three parts

$$
\alpha U(s(t), a(t))+\beta U(r(t), w(t))+U(q(t))
$$

where individuals give higher weight to the utility from gambling $(\alpha>\beta)$ and as in Dragone (2015), $U(q(t))=q(t)$ for simplicity. $U(s(t), a(t))$ and $U(r(t), w(t))$ are assumed to be continuously differentiable, with negative second order derivatives $\left(U_{s s}, U_{a a}, U_{r r}, U_{w W}<0\right)$. For simplicity and without lack of generality 
preferences for gambling are incorporated in the $U_{s}>0$ assumption.

Following Dragone et al. [17], we define reinforcement the case where, the more a person is addicted to gambling, the more she desires to consume the addictive good, so that the marginal utility of consuming an addictive good increases with past consumption of that good, that is, $U_{s a}>0$. On the other side, tolerance means that utility from a given amount of consumption is lower when past consumption is greater, implying that $U_{a}<0$ for $a>0$.

We also assume that $r$ displays "virtuous" reinforcement effects in well-being accumulation, hence $U_{r W}>0$.

In a continuous-time infinite horizon optimization problem, the individual chooses the optimal levels of $s$ and $r$, by solving the following intertemporal problem

$$
\begin{aligned}
& \max _{s(t), r(t), q(t)}\left\{\alpha\left[\int_{0}^{\infty} \mathrm{e}^{-\rho t} U(s(t), a(t)) \mathrm{d} t\right]\right. \\
& \left.+\beta\left[\int_{0}^{\infty} \mathrm{e}^{-\rho t} U(r(t), w(t)) \mathrm{d} t\right]+\left[\int_{0}^{\infty} \mathrm{e}^{-\rho t} U(q(t)) \mathrm{d} t\right]\right\}
\end{aligned}
$$

s.t.

$$
\begin{gathered}
M=q(t)+p_{s} s(t) \\
\dot{a}(t)=s(t)-\delta a(t), a(0)=a_{0} \\
\dot{w}(t)=r(t)-\delta w(t)-\varepsilon s(t), w(0)=w
\end{gathered}
$$

Equation (2) is the usual budget constraint where $M$ represents the individual's income (that we assume is the only source of wealth) and $p_{s}$ is the price to partecipate to game $s$.

The differential Equations (3) and (4) represent the stock and flow dynamics related to the two goods. In essence, the difference between the two are that, while the effect of accumulation of $r$ produces a utility, the effect of addiction produces per se both a disutility and a reinforcement of the utility arising from further consumption for $a$.

For simplicity, omitting the time index, we obtain the following current-value Hamiltoniian function and the related to first order conditions on the state and costate variables:

$$
\begin{gathered}
H=\alpha U(s, a)+\beta U(r, w)+M-p_{s} s+\mu(s-\delta a)+\lambda(r-\varepsilon s-\delta w) \\
\beta U_{r}=-\lambda \\
\alpha U_{s}-p_{s}=-\mu+\lambda \varepsilon \\
\dot{\mu}=(\rho+\delta) \mu-\alpha U_{a} \\
\dot{\lambda}=(\rho+\delta) \lambda-\beta U_{w}
\end{gathered}
$$

and the transversality condition:

$$
\lim _{t \rightarrow \infty} \mathrm{e}^{-\rho t}[\mu(t) a(t)+\lambda(t) r(t)]=0
$$

Differentiating Equations (5) and (6) with respect to time $t$, after some 
manipulations the previous system in steady-state reduces to:

$$
\begin{gathered}
-(\rho+\delta) U_{r}=U_{w} \\
U_{a}=\frac{1}{\alpha}\left\{(\rho+\delta)\left(p_{s}-\alpha U_{s}\right)+\varepsilon \beta U_{w}\right\} \\
s(t)=\delta a(t) \\
r(t)=\delta w(t)-\varepsilon s(t)
\end{gathered}
$$

Condition (10) states that the marginal utility of addiction is equal to a weighted sum of the utility from gambling and utility from wellbeing arising from the ability generated by consumption of the $r$. Finally, conditions (11) and (12) obviously derive from the lack of accumulation/depletion of both addiction and wellbeing from the consumption of $r$ in steady state. From conditions (9)-(12) it is also clear that an agent rationally converges to a stable situation where both the well-being is maximized and she is not addicted to gambling $\left(U_{a}=U_{w}=0\right)$ in steady state, only when $U_{s}=p_{s} / a$ is positive, when the cost of gambling is equal to the marginal utility of gambling.

Definition: A Pathological Gambler is an individual aware that $\mathrm{U}_{\mathrm{a}}<0$, but nonetheless choosing an amount of gambling leading to that harmful addiction level under the condition

$$
\beta<\frac{(\rho+\delta)\left(p_{s}-\alpha U_{s}\right)}{\varepsilon U_{w}}
$$

We substantially define as pathological gambler an individual that, even by knowing that gambling addiction is harmful (since $U_{a}<0$ by assumption) nonetheless increases addiction by gambling more and choosing low levels of $r$ since she gives high weight to the utility from gambling and low weight to the utility from the enjoyment of the stimulus good, or, more specifically,

$\beta<\frac{(\rho+\delta)\left(p_{s}-\alpha U_{s}\right)}{\varepsilon U_{w}}$.

From (9)-(12) we find that there are three feasible equilibria satisfying the assumption $U_{a}<0$.

Case 1): assuming that $(\rho+\delta)\left(p_{s}-\alpha U_{s}\right)>0$, and, as a consequence $U_{s}<0<p_{s} / a$. Since $U_{a}<0$ by assumption, the second part of the sum in (10) has to be negative implying that $U_{w}$ is also negative. This means that, by (12), $U_{r}>0$ and therefore, as a consequence, the individual is below the maximum level of consumption of $r$. A second implication is that the second part of the (12) must not just be negative but also higher than the first positive part. This is possible if (13) is satisfied. Case 1) may be defined as the case of a Pathological Gambler which consumes high quantities of $s$ and low quantities of $r$.

Other two possible equilibria are:

Case 2) since $U_{a}<0$, we may assume that the first part of the sum in (12) is negative. This implies that $(\rho+\delta)\left(p_{s}-\alpha U_{s}\right)<0$, or $U_{s}>\left(p_{s} / a\right)>0$, and $U_{w}$ $<0$, which implies from (10) that $U_{r}>0$. In this case the individual is consuming 
below the maximum levels of both goods $s$ and $r$.

Case 3) again from $U_{a}<0$, we may have that the first part of the sum of (10) is negative and therefore $U_{s}>\left(p_{s} / a\right)>0$, while $U_{w}>0$. In this case the individual consumes low quantities of $\mathrm{s}$ and high quantities of $r$. This case is satisfied by high levels of $\beta$. Note as well that, the higher $\beta$, the more difficult is to satisfy $U_{a}$ $<0$. This may be considered the case of a "mindful" or "conscious" gambler.

Notice that in the pathological gambler equilibrium (Case 1) we have in steady state that $s=\delta a$, that is, the consumption of $s$ is high enough to compensate the decay of the consumption habit. Hence the process reducing dependence does not start and this players remain a pathological gambler.

It is possible to show that (9)-(12) represent a not uncommon case of stable steady state solutions for high enough values of $\alpha$ versus $\beta$, corresponding to a stable saddle point, according to conditions for a modified Hamiltonian System as in Dockner and Feichtinger [18], especially when we assume a quadratic specification which satisfies the assumptions made for the utility function and which allows for closed-form solutions (i.e., for instance, by assuming $U(s(t), a(t))=s\left(x a-\frac{s}{2}\right)-\frac{a^{2}}{2}$ and $\left.U(r(t), w(t))=r\left(y w-\frac{r}{2}\right)-\frac{w^{2}}{2}\right)$. Full details on results from this specific functional form are available upon request).

The intuition behind Case 1 is that the individual overconsumes gambling beyond its maximum in terms of partial marginal utility due to the reinforcement effect $\left(U_{s a}>0\right)$ which compensates the direct utility loss from addiction $\left(U_{a}<0\right)$ and the missed utility arising from under consumption of $r$. This may occur only for very low utility weights given to this last ( $\beta$ below a certain threshold). The negative side of pathological gambling therefore lies in the consumption of something which produces per se utility (the good $s$ ) but however generates a side effect (addiction) which produces a disutility to the same individual $\left(U_{a}<0\right)$. The net utility from the two effects remains however positive. Note that, even though we may think that it is not "healthy" to maximize in this way (due to addiction growth) the Pathological Gambler is still rational and maximising her "total" utility (where for total we mean the sum of its three parts). Why therefore we should stigmatize its choice? If instead of gambling we would examine smoking we could assume that the pathological smoker may underestimate the health costs of smoking. Again this should be a form of irrationality (due to the incapacity of incorporating such costs in her utility function).

\section{The Social Costs of Pathological Gambling}

Beyond the effect on the gambler itself, the simplest way to model the social costs of Pathological Gambling is by assuming, as we do, that the Pathological Gambler is part of a household and that her behavior has negative externalities on the household budget and relations. The simplest way to model it is to consider the presence of a second "passive" member (since now on pm), i.e. an 
individual in the household who suffer from the consequences of gambling of the other individuals. In fact, under the Pathological Gambling scenario money available for consumption of the numerary good is reduced and this has negative effects on the passive household member. We may in addition and reasonably believe $r$ is a relational good which is also consumed jointly with passive household members. Also in this case under consumption of gambling has direct negative consequences on the wellbeing of the other household members. The point we make highlights an important issue: Pathological Gambling becomes suboptimal in presence of rational and maximizing gamblers when we consider their relational ties with other individuals who are negatively affected by their behavior. To take into account these possibilities we make some simplifying assumptions.

First of all we assume that only two individuals exist in a given household, that is mindful/conscious and pathological players. We assume that the conscious player attach to the utility of gambling the weight $\alpha_{p m}$ which can also be null if he doesn't like the gambling at all, while he found that more important are the relational goods, so that his $\beta_{p m}$ is higher than the threshold found is Section 2.

The conscious player solves:

$$
\begin{aligned}
& \max _{s(t), r(t), q(t)}\left\{\alpha_{p m}\left[\int_{0}^{\infty} \mathrm{e}^{-\rho t} U(s(t), a(t)) \mathrm{d} t\right]\right. \\
& \left.+\beta_{p m}\left[\int_{0}^{\infty} \mathrm{e}^{-\rho t} U(r(t), w(t)) \mathrm{d} t\right]+\left[\int_{0}^{\infty} \mathrm{e}^{-\rho t} U\left(q_{p m}(t)\right) \mathrm{d} t\right]\right\}
\end{aligned}
$$

Under the same constraints (2)-(4).

If we consider the optimal level of gambling $s_{p m}$ which maximizes the utility function of a conscious gambler (which would be zero if the conscious liked playing at all), and define the corresponding optimal value function $V_{o}^{*}=V\left(s_{p m}^{*}, r_{p m}^{*}, q_{p m}^{*}\right)$. When damaged by the behavior of the other individual who is a Pathological Gambler, his optimal value function $V_{l}^{*}$ is found by solving the new "passive" member problem which is equal to (1) but where now the utility of the passive member is reduced both terms for a share $k$ proportionally to the greater devotion that the other gives to gambling rather than taking care of the family $\alpha-\alpha_{p m}$, and in terms of the quantity of the numeraire good, that he can consume after having paid gambling and consumption for the PG, $q_{p m}=M_{p m}-p_{s} s^{p m}-p_{s} s^{*}-q$, where $s^{*}$ is the optimum for the PG and $q$ is the minimum quantity of necessary good for a PG that the $p_{m}$ will support for him. We are assuming that PG can consume $q=M-$ $p_{s} s$ if he has his own monetary income $M>0$, and a positive share of the other's income otherwise. In other words, the PG has no income he can still consume at least a minimum necessary share $q$ and the desired $s$ at a price $p_{s} 8$ if he is borrowing money by using the available income from the $p m$, who on turn should take as given the PG decision and maximize his consumption of $q_{p m}$ as if he has only a limited amount of available income which remains afer having paid $q$ and $a$. 
If in this case as it is reasonable to assume he would prefer not to play at all to consume more $q_{p m}$ then the loss that would result affect not only in the relational goods $r$ and $q$ but also his consumption of $s^{p m}$ being conscious that can not consume anymore.

Anyway by considering only the most relevant social costs which a Social Planner should take into account, the passive member Welfare Loss may be measured by:

$$
L_{p m}=V_{o}^{*}-V_{l}^{*}=\alpha k U(r)+p_{s} s+q
$$

\section{How to Discourage the Pathological Gambling: Two Alternatives for the Government's Behaviour}

In this section we argue that the government may use two different strategies in order to tackle gambling addiction:

- Increasing the price, $p_{s}$ through higher taxes, which would represent for the gambler a permanent expected decrease in her wealth to take into account in her maximization problem.

- Directly reduce the amount of available gambling opportunities (for instance by banning slot machines in the stores or creating limits to the maximum amount of bets from a given machine or related to a given individual) which implies setting up a maximum amount: $0 \leq s<\underline{s}$.

1) We first consider the policy aimed at directly affecting the cost of gambling by raising its price, for instance through the introduction of excise taxes on sellers and we analyze its long-run effects by focusing on stable steady states. Our results, by following Becker and Murphy [5], show that when the price of gambling increases, gamblers addicted do respond to incentives and in the long-run they reduce the consumption of the addictive good when it becomes more expensive since

$$
\frac{\partial s^{s s}}{\partial p_{s}}<0
$$

where $s^{s s}$ denotes the value of $s$ in steady-state.

2) We model the government taking into account its trade-off in dealing with the gambling problem. On one side, the government earns revenues from gambling activities while, on the other side, it may be concerned about the negative social externalities that gambling generates. More specifically, we assume that a myopic government want to earn a minimum amount of revenues from gambling, with revenues which cannot fall below the level $\underline{E}$.

$$
E=\gamma p_{s} s \geq \underline{E}
$$

where $\gamma$ is the tax rate calculated as a percent amount of the price $p_{s}$ paid on each gambling play.

In this case the minimum gambling opportunities fixed by the myopic government, which want to follow policy 2 and respect its minimum revenue constraint, cannot be lower than 


$$
\underline{s} \geq \frac{\underline{E}}{\gamma p_{s}}(\text { policy } 2 \mathrm{a})
$$

On the contrary, for a non myopic government being aware of the trade-o between gambling revenues and social externalities from gambling (which produce a level of welfare loos measured by Loss $_{p m}$ ), the previous constraint (in case of decision to follow policy 2) becomes a policy $2 \mathrm{~b}$ :

$$
\gamma p_{s} \underline{s}+\theta\left(\underline{w}-w^{s s}\right) \geq \underline{E}
$$

where $w^{s s}$ corresponds to the steady-state solution.

Therefore we have a more stringent threshold and the government which takes into account the trade-off will fix a lower share of maximum gambling allowance.

$$
s \geq \frac{\underline{E}-\theta\left(\underline{w}-w^{s s}\right)}{\gamma p_{s}} \text { policy } 2 \mathrm{~b}
$$

What may determine this trade-off is that, on the one side, the government cares about tax revenues while, on the other side, it considers that the sensitiveness of the public opinion to the social costs of gambling may affect its re-election.

In what follows we aim to compare:

- the differences between a far-sighted government and a

short-sighted/self-interested government.

- the most efficient policy between 1) and policy 2b).

1) About the reduction in the consumption of $s$ in steady state, when the government fixes a limit s, under policy 1, by using (19) and replacing steady the state level of $s^{*}$ we have that

$$
\underline{s} \text { iff } \delta a^{s s}>\frac{\underline{E}}{\gamma p_{s}}
$$

The greater the addiction of the PG in equilibrium $\left(a^{*}\right)$, the higher is the gambling consumption chosen by the PG in steady state $\left(s^{\star}\right)$. Hence, if the government fixes a minimum amount of gambling revenues $E$ at high levels, there could not be too much difference between the implicit maximum level of gambling allowed by the government and that desired by the PG. The latter is obviously much lower, the higher the price. In the second case is much more likely that the restriction leads to a level of $s$ certainly lower than the desired one, because

$$
s^{s s}>\underline{s} \text { iff } \delta a^{s s}>\frac{\underline{E}-\theta\left(\underline{w}-w^{s s}\right)}{\gamma p_{s}}
$$

To provide an example of the trade-off in practice, in December 2013 the Italian parliament voted an amendment to the Financial law where local administrations which enforced laws limiting gambling activities were asked to compensate the reduced scale revenues for the government budget. The bottom-up pressure from media and the public opinion forced the government 
to withdraw the amendment. Another example is the city of New York where in November the 5th, 2013 the council directly investigated citizens preferences with a referendum on the creation of new video lotteries.

What matters when fixing the threshold is the level of $L_{p m}$ (a factor which impacts negatively on the threshold) that a society considers important to preserve, the minimum amount of revenue $E$ that the government aims to achieve (which positively affects the threshold) and the coefficients $\gamma$ and $\delta$ which reduce the threshold because they increase the entries even when gambling is strongly limited.

2) We must now compare policy 1 versus $2 b$. When the government opts for a price increase, the last policy is more efficient, in terms of amount of gambling reduced, holding the same revenues $\underline{E}$.

Specifically, the last policy $2 \mathrm{~b}$ is more efficient and implies a higher reduction of gambling if

$$
\delta a^{s s}\left(1-\frac{\partial s^{s s}}{\partial p_{s}}\right)-\delta a^{s s}+\frac{\underline{E}-\theta\left(\underline{w}-w^{s s}\right)}{\gamma p_{s}}<0
$$

Or

$$
\frac{\left[E-\theta\left(\alpha K U(r)+p_{s} \delta a^{s s}+q\right)\right]}{\gamma p_{s}}<\delta a^{s s} \frac{\partial s}{\partial p_{s}}
$$

Therefore if the government is following a policy of type (2b) the last condition is certainly verified for high value of $L_{p m}$ and therefore for high value of the Addiction. In fact for high values the passive member attaches to any additional unit of $r$ instead of $s$ with respect to the PG, i.e. his $k U_{(r)}$ which will be lower for the PG as he devotes more care to gambling, and for higher values of the mimimum amount $q$ the PG needs to consume and that he can't pay by himself. In this case a policy of type (2b) leads to a greater disincentive to the PG, so reducing losses for the passive member and let the government saving money to support this losses. The intuition behind our result is that gamblers' dependence reduces the effectiveness of higher prices vis-à-vis outright gambling limitations. The addicted gambler will try to gamble even if $p_{s}$ is higher and the price increase may produce the paradox of further reducing wellbeing of passive household members without limiting gambling consumption. This result is in agreement with recent empirical analysis on the effects of restrictions policies on another kind of addiction, i.e. smoking addiction, Wakefield et al. [19] determine the relation between extent of restrictions on smoking at home, at school, and in public places and smoking prevalence among school students. Their results suggest that more extensive bans on smoking in public places, and enforced bans on smoking at school may reduce teenage smoking. According to Farrelly et al. [20] having a 100\% smoke-free workplace would reduce smoking prevalence by $6 \%$ points and average daily consumption among smokers by $14 \%$ relative to workers subject to minimal or no restrictions. Fichtenberg and Glantz [21] compare the effects of restriction policies with those of an increase in excise 
taxes and find that smoke-free workplaces not only protect non-smokers from the dangers of passive smoking, but also reduce consumption in more significant proportion. To obtain the same effect sigarette taxation should increase by $300 \%$.

In that follows we argue that a contribution to solve the gambling problem may be found by devising "bottom-up" initiatives by which "socially responsible citizens" contribute with their action to produce "nudging" effects on myopic governments. In essence, the bottom up action of the public opinion may modify the government perception of the parameter $\theta$, thereby a ecting its trade-o and leading to a stronger enhagement of the latter against gambling. There are several works [21] [22] showing how a small share of consumer choices affected by social and environmental concerns significantly influence the behaviour of profit maximizing firms whose economic and financial success depend on small changes in market shares, revenues and pro ts. Institutions are nonetheless affected as politicians try to represent issues of these groups not to lose their political support.

One of these important bottom-up approaches which has been recently experienced is that of the so-called "slot-mobs"

(http://www.nexteconomia.org/slots) - mob. The idea is to create the largest possible group of citizens and bring them to reward with their shopping actions cafeterias which made the choice to remove slot machines from their shops. Since 2013 a coalition of over 70 organizations in Italy organized slot-mobs in several Italian cities with the goal of raising awareness of the population against the risk of gambling. After the beginning of the slot mob initiative several laws restricting gambling have been taken in the cities in which slot-mobs were operated. What may be inferred is that slot-mobs have raised the sensitivity of voters on the issue and thereby increased the relevance of the parameter $\theta$ in (17), leading the government to give relatively more weight to the social costs of gambling than to its tax revenues.

\section{Conclusion}

Our paper relates to the economic literature of addiction by addressing the novel, and less thoroughly explored, issue of gambling addiction. In an intertemporal continuous time model we define pathological gambling and discuss conditions under which pathological gambling, even representing the optimal choice for the gambler in terms of aggregate utility, which produces "painful" addiction for her plus social costs. Based on this theoretical framework we further address the issue of the optimal policies needed to tackle this phenomenon. In our model the pathological gambler is a rational and maximizing individual who compensates the disutility arising from increasing addiction with the utility of gambling which is enhanced by the reinforcement effect of past gambling choices. Our model however provides a theoretical framework which properly takes into account social costs of pathological gambling. The pathological gambler is part of a household composed by a second "passive" member whose utility depends on the other two goods 
available to her/him and the pathological gambler (a standard numerary consumption good and a complimentary relational good). Consumption of the above mentioned two goods is however reduced by the pathological gambler's over-consumption of gambling. A long sighted government may act on the problem in two ways. First, it may increase the cost of gambling thereby reducing gambling consumption for the pathological gambler. Second, it may directly reduce gambling opportunities. In our model we demonstrate why and under what conditions the second solution is to be preferred. We also discuss the role played by bottom up action of a minority of "gambling concerned" individuals (as occurred with the recent wave of slot-mobs) which can raise government sensitivity on the issue; thereby a ecting the trade-o of the latter between tax revenues from gambling and gambling restrictions. Our theoretical framework may be a benchmark for future research on the issue. In our model we demonstrate that the pathological gambling equilibrium occurs only when the individual attributes extra weight to gambling activity. Since it is reasonable to assume that ads on gambling may significantly affect such preference weight, a third policy action of banning gambling ads may be effective in prevent pathological gambling. Also mixed policy options affecting both sides of the trade-off such as those of taxing gambling ads could be further explored.

\section{Conflicts of Interest}

The authors declare no conflicts of interest regarding the publication of this paper.

\section{References}

[1] Potenza, M.N. (2008) Review. The Neurobiology of Pathological Gambling and Drug Addiction: An Overview and New Findings. In: Robbins, T.W., Everitt, B.J. and David Nutt, D.J., Eds., Neurobiology of Addiction: New Vistas, Royal Society Publishing, Oxford, 43-76.

[2] Ross, D., Kincaid, H., Spurrett, D. and Collins, P. (2010) Economics Models of Pathological Gambling, in What is Addiction? MIT Press, Cambridge. https://doi.org/10.7551/mitpress/9780262513111.003.0006

[3] Stigler, G.J. and Becker, G.S. (1977) De gustibus non est disputandum. American Economics Review, 67, 76-90.

[4] Iannaccone, LR. (1986) Addiction and Satiation. Economic Letters, 21, 95-99. https://doi.org/10.1016/0165-1765(86)90130-8

[5] Becker, G.S. and Murphy, K.M. (1988) A Theory of Rational Addiction. Journal of Political Economy, 96, 675-700. https://doi.org/10.1086/261558

[6] Gul, F. and Pesendorfer, W. (2001) Temptation and Self-Control. Econometrica, 69, 1403-1435. https://doi.org/10.1111/1468-0262.00252

[7] Walker, D. (2008) Clarification of the Social Costs of Gambling. Journal of Public Budgeting, Accounting, and Financial Management, 20, 141-152. https://doi.org/10.1108/JPBAFM-20-02-2008-B001

[8] Bardsley, P. (1998) Dynamic Addiction Games. Working Papers Series 632, Department of Economics, The University of Melbourne, Melbourne. 
[9] Hartley, R. and Farrell, L. (2002) Can Expected Utility Theory Explain Gambling? The American Economic Review, 92, 613-624.

[10] Walker, D. and Jackson, J. (2011) The Effect of Legalized Gambling on State government Revenue. Contemporary Economic Policy, 29, 101-114. https://doi.org/10.1111/j.1465-7287.2010.00198.x

[11] Layton, A. and Worthington, A. (1999) The Impact of Socio-Economic Factors on Gambling Expenditure. International Journal of Social Economics, 26, 430-440. https://doi.org/10.1108/03068299910230035

[12] Szakmary, A. and Szakmary, C.M. (1995) State Lotteries as a Source of Revenue: A Re-Examination. Southern Economic Journal, 56, 1167-1181. https://doi.org/10.2307/1060748

[13] Madhusudhan, R.G. (1996) Betting on Casino Revenues: Lessons from State Experiences. National Tax Journal, 49, 401-412.

[14] Rivenbark, W.C. and Rounsaville, B.B. (1996) The Incidence of Casino Gaming Taxes in Mississippi: Setting the Stage, Public Administration Quarterly, 20, 129-142.

[15] Kearney, M. (2005) State Lotteries and Consumer Behavior. Journal of Public Economics, 89, 2269-2299. https://doi.org/10.1016/j.jpubeco.2004.07.004

[16] Mikesell, J. and Pirog-Good, M. (1990) State Lotteries and Crime. American Journal of Economics and Sociology, 49, 7-20. https://doi.org/10.1111/j.1536-7150.1990.tb02254.x

[17] Dragone, D., Manaresi, F. and Savorelli, L. (2016) Obesity and Smoking: Can We Catch Two Birds with One Tax? Health Economics, 25, 1464-1482.

https://doi.org/10.1002/hec.3231

[18] Dockner, E.J. and Feichtinger, G. (1991) On the Optimality of Limit Cycles in Dynamic Economic Systems. Journal of Economics, 53, 31-60. https://doi.org/10.1007/BF01227014

[19] Wakefield, M., Chaloupka, F., Kaufman, N.C., Tracy,C., Barker, D.C. and Ruel, E.E. (2000) Effect of Restrictions on Smoking at Home, at School, and in Public Places on Teenage Smoking: Cross Sectional Study. British Medical Journal, 321, 321-333. https://doi.org/10.1136/bmj.321.7257.333

[20] Farrelly, M.C., Evans, W.N. and Sfekas, A.E. (1999) The Impact of Workplace Smoking Bans: Results from a National Survey. Tobacco Control, 8, 272-277. https://doi.org/10.1136/tc.8.3.272

[21] Becchetti, L., Palestini, A., Solferino, N. and Tessitore, M.E. (2014) The Socially Responsible Choice in a Duopolistic Market: A Dynamic Model of Ethical Product Differentiation. Economic Modelling, 43, 114-123. https://doi.org/10.1016/j.econmod.2014.07.039

[22] Becchetti, L., Federico, G. and Solferino, N. (2011) What to Do in Globalised Economies if Global Governance Is Missing? The Vicarious Role of Competition in Social Responsibility, Competition in Social Responsibility. International Review of Economics, 58, 185-211. https://doi.org/10.1007/s12232-011-0122-0 